\title{
Concomitant Heroin and Cocaine Use among Opioid-Dependent Patients during Methadone, Buprenorphine or Morphine Opioid Agonist Therapy
}

Gastberger, Salome ; Baumgartner, Markus R ; Soyka, Michael ; Quednow, Boris B ; Hulka, Lea M ; Herdener, Marcus ; Seifritz, Erich ; Mutschler, Jochen

DOI: https://doi.org/10.1159/000500542

Posted at the Zurich Open Repository and Archive, University of Zurich ZORA URL: https://doi.org/10.5167/uzh-176180

Journal Article

Published Version

Originally published at:

Gastberger, Salome; Baumgartner, Markus R; Soyka, Michael; Quednow, Boris B; Hulka, Lea M; Herdener, Marcus; Seifritz, Erich; Mutschler, Jochen (2019). Concomitant Heroin and Cocaine Use among Opioid-Dependent Patients during Methadone, Buprenorphine or Morphine Opioid Agonist Therapy. European Addiction Research, 25(4):207-212.

DOI: https://doi.org/10.1159/000500542 


\title{
Concomitant Heroin and Cocaine Use among Opioid-Dependent Patients during Methadone, Buprenorphine or Morphine Opioid Agonist Therapy
}

\author{
Salome Gastberger ${ }^{a}$ Markus R. Baumgartner ${ }^{b}$ Michael Soyka ${ }^{c, g}$ \\ Boris B. Quednow ${ }^{d}$ Lea M. Hulka ${ }^{a}$ Marcus Herdener ${ }^{a}$ Erich Seifritz ${ }^{e}$ \\ Jochen Mutschler ${ }^{a}, f$ \\ a Department of Psychiatry, Center for Addictive Disorders, Psychotherapy and Psychosomatics, Psychiatric Hospital, \\ University of Zurich, Zürich, Switzerland; b Zurich Institute of Forensic Medicine, Center for Forensic Hair Analysis, \\ University of Zurich, Zürich, Switzerland; ' ${ }^{c}$ Medical Park Chiemseeblick Fachklinik für Psychosomatik, Bernau, Germany; \\ ${ }^{\mathrm{d}}$ Department of Psychiatry, Experimental and Clinical Pharmacopsychology, Psychotherapy and Psychosomatics, \\ Psychiatric Hospital, University of Zurich, Zürich, Switzerland; ${ }^{e}$ Department of Psychiatry, Psychotherapy and \\ Psychosomatics, Psychiatric Hospital, University of Zurich, Zürich, Switzerland; ${ }^{f}$ Privatklinik Meiringen, Postfach 612, \\ Meiringen, Switzerland; ${ }^{9}$ Psychiatric Hospital University of Munich, Munich, Germany
}

\section{Keywords}

Methadone $\cdot$ Buprenorphine $\cdot$ Morphine $\cdot$ Craving $\cdot$ Opioid dependency · Stimulants

\footnotetext{
Abstract

Background: Among all the treatment methods developed so far, opioid agonist treatment (OAT) is the most effective therapy for opioid dependence. While methadone (MTD) is the most commonly used, fewer data are available on alternative opioid agonist. The aim of this study was to assess the efficacy of buprenorphine (BUP) and slow-released morphine compared to MTD with regard to the reduction of concomitant heroin and cocaine use. Methods: This cross-sectional study included 105 patients receiving MTD, BUP, or slow-release morphine as opioid agonist therapy at the Psychiatric Hospital of Zurich. Illicit drug use was assessed using a retrospective 3-month hair toxicology analysis to quantify concentrations of heroin degradation products and metabo-
}

lites, as well as cocaine and cocaine metabolites. We have also collected self-reports, but in the data of the study, only the results of the hair analysis were considered. Results: BUP-treated patients showed lower rates of illicit opiate consumption in comparison to the group treated with MTD or slow-released morphine $(p<0.05)$. The proportion of heroinpositive hair samples associated with slow-release morphine treatment was similar to the proportion associated with MTD treatment. Neither the MTD vs. slow-released morphine groups nor the BUP vs. MTD groups showed significant differences in the number of patients consuming cocaine although patients in the BUP group had significantly lower concentrations of cocaine in hair testing compared to the patients in the MTD group. Prevalence of cocaine consumption was also significantly lower in the BUP group compared to patients in the slow-release morphine group $(p<0.05)$. Conclusion: This study suggests that BUP OAT is associated with reduced additional opiate co-use.

(c) 2019 S. Karger AG, Base

\section{KARGER}

(C) 2019 S. Karger AG, Basel

E-Mail karger@karger.com

www.karger.com/ear
Salome Gastberger

Department of Psychiatry, Center for Addictive Disorders

Psychotherapy and Psychosomatics, Psychiatric Hospital, University of Zurich

Selnaustrasse 9, CH-8001 Zürich (Switzerland)

E-Mail salome.gastberger@uzh.ch 


\section{Introduction}

Opioid dependence is a chronic relapsing disorder with important public health implications, and affects about 15.6 million patients worldwide [1]. One of the most effective therapies for opioid dependence is opioid agonist treatment (OAT) [1]. Together with psychological support, this treatment stabilizes opioid-dependent patients and reduces illicit opioid use, infectious-disease transmission, and death due to overdose [2,3]. Some studies not only observed an effect of OAT on heroin use, but also reported on a significant reduction of concomitant cocaine use $[4,5]$. Further benefits include improved quality of life and improved social reintegration [6]. The most commonly prescribed medication in OAT Switzerland and Europa is methadone (MTD) [6,7]. MTD was the first and is now the most established OAT medication, with its effectiveness proven in many studies [8]. However, there are patients who do not tolerate MTD or have an inadequate withdrawal suppression often associated with high illicit opioid co-consumption [9]. In addition, some specific side effects of MTD influence compliance and limit its clinical usefulness, such as weight gain, sweating, depressive symptoms, loss of libido, QT-prolongation and a potential for interactions with other medications [10].

In Europe, other treatment options such as buprenorphine (BUP) and slow-release oral morphine (SROM) are available. BUP is a partial $\mu$-opiate agonist and $\kappa$-opiate antagonist with established efficacy in the treatment of opiate dependence. As BUP undergoes extensive firstpass metabolism it has to be taken sublingually [11]. BUP has some advantages over MTD as it is less pro-arrhythmic, carries a lower risk of respiratory depression when overdosed, and has a longer duration of action that allows dispensing at intervals of 2-3 days [12]. Studies have shown that BUP leads to significantly lower consumption of illicit opioids $[7,13,14]$, and also seems to reduce concomitant cocaine use [15]. In subjects affected by depression and mood disorders in particular, BUP reduced rates of illicit opiate consumption [16].

Morphine is a full $\mu$-opioid receptor agonist. Once-aday SROM is an approved medication with proven efficacy and safety for the treatment of opioid dependence $[17,18]$. SROM may result in reduced opioid craving and improved tolerability compared to MTD $[9,19]$, and is at least as effective as MTD in reducing illicit opioid consumption [20].

Familiarity with effective alternatives to MTD is not universal among psychiatrists, as shown in a recent study from Switzerland reporting that $17 \%$ of treating psychia- trists were unaware of the existence of effective treatments other than MTD [7]. It is important that psychiatrists are aware of the existence of alternative medications for OAT in order to optimize and individualize therapy. Alternative medication may sometimes have a better effect on withdrawal symptoms, craving behaviour and the reduction of illicit opioid co-use.

The aim of this study was to evaluate the effectiveness of MTD, BUP and SROM with regard to reduction of heroin and/or cocaine co-use in opioid-dependent patients in OAT. We hypothesized that BUP and slow-release morphine would be as effective or superior to MTD maintenance treatment in terms of the outcome measures illicit opioid and cocaine use.

\section{Materials and Methods}

\section{Subjects}

The study was conducted in 2014 in an outpatient setting at the Centre for Addictive Disorder of the Psychiatric Hospital Zurich [21]. OATs in Switzerland are covered by mandatory health insurance. Every patient who is diagnosed with heroin dependence is accepted for treatment and there is no waiting time. The choice of the appropriate OAT medication depends mainly upon patients and providers rather than regulatory constraints [22]. The treatment can be provided in specialised outpatient centres or private practices. All patients who were in OAT at the time of study execution with either MTD, BUP or SROM were invited to participate in the study, and 105 patients (28 females, 77 males; mean age $41.2 \pm 8.8$ years) were enrolled. The inclusion criterion for participation was the diagnosis of opioid dependence according to the DSM-IV criteria. The exclusion criteria were age under 18 and over 65 years and insufficient German language skills. Patients with psychiatric comorbidities or prescribed medication other than opioid substation were not excluded.

\section{Study Design}

In this cross-sectional study, all patients in OAT were recruited to participate in the study when they came to collect their opioid agonist medication. Participants were then categorized into 3 treatment groups depending on the opioid agonist medication currently taken. Demographic data such as age, gender, years and type of education, employment status, number of clinical treatments, as well as medication and self-report drug use, were assessed with self-reported questionnaires. Data on psychiatric diagnosis at the time of study recruitment were collected from electronic medical records of the patients. Depression and anxiety were assessed using the Beck Depression Inventory [23] and StateTrait Anxiety Inventory [24]. Craving was assessed with a Visual Analog Scale ranging from 1 to 100) [25].

\section{Study Assessment}

The primary outcome was the prevalence of concomitant heroin (6-monoacetylmorphine [MAM]) and cocaine use detected by means of 3-month hair toxicology analysis (liquid chromatogra- 
phy-tandem mass spectrometry) [26]. A secondary goal was to determine the concentration of heroin and cocaine in the hair samples and to identify possible factors such as the substitution dose or craving behaviour.

\section{Hair Analysis}

The measure for assessing illicit drug use was a 3-month hair toxicology analysis, which was carried out at the Institute of Forensic Medicine at the University of Zurich. Whenever possible head hair was used, but body hair was used in cases where little or no, or only heavily bleached hair was available. In the hair analysis, only the proximal $3-\mathrm{cm}$ section of the collected head hair locks was investigated. Assuming an average growth rate of $1 \mathrm{~cm} / \mathrm{months}$, this hair segment represents the consumption behaviour of the last 3-4 months before collection. The analytical limits of the method enable the detection of very low concentration in the hair samples. However, strong cosmetic treatments like bleaching can degrade the incorporated substances [27]. Hair was analysed according to Rust et al. [28]. The hair sample was washed in water, acetone and hexane for $2 \mathrm{~min}$ each. Then it was dried at room temperature, cut into small snippets and extracted in methanol (extraction step 1) and a mixture of methanol and ammonium formate buffer (extraction step 2) for 90 min each. After each extraction step, the organic layers were separated by centrifugation and dried under a stream of nitrogen at $36^{\circ} \mathrm{C}$. Finally, the residues were combined, redissolved in methanol and aqueous ammonium format buffer solution and then the samples were analysed with LC-MS/MS. Positive heroin and cocaine use was confirmed by the presence of MAM or cocaine + cocaethylene, respectively, using cut-off values of $15 \mathrm{pg} / \mathrm{mg}$ MAM and $60 \mathrm{pg} / \mathrm{mg}$ cocaine + cocaethylene. These low threshold values discriminate between the abstinence and occasional use of drugs.

\section{Statistical Analysis}

Data analysis was carried out using SPSS 24 (SPSS, Chicago, IL, USA). Differences in baseline characteristics between treatment groups were compared by Student $t$ tests for continuous variables and chi-square tests for categorical variables. In order to compare the 3 OAT groups for additional heroin and/or cocaine consumption, we analysed heroin and cocaine hair concentrations using the Kruskal-Wallis-Test for non-parametric variables. Normal distribution was tested with Kolmogorov-Smirnov Test. To determine the percentage of patients with additional heroin or cocaine consumption, we recoded the results of the hair analysis, classifying all values $\geq 1$ as yes and all values $=0$ as no, and then compared the substitution groups using the Pearson chi-square test. To detect correlations between substitution dose, craving and drug consumption we used the two-tailed Spearman-Rho correlation test after conducting a log-transformation of the hair analysis data. $p$ values $<0.05$ were considered statistically significant.

\section{Results}

\section{Demographic Characteristics}

We recruited 110 opioid-addicted patients in opioid agonist therapy. Because of missing data due to incom- plete questionnaire and medical record data, 5 patients had to be excluded. Many subjects had comorbidities, with over $80 \%$ being diagnosed with at least one other psychiatric disorder in addition to the opioid dependence, and nearly half reporting a diagnosis of hepatitis C (41.2\%). There were no significant differences between the 3 substitution groups in age, gender, years and type of education, employment status, number of clinical treatments, number of psychiatric comorbidities, BDI score or the frequency of occurrence of HIV or Hepatitis C. Detailed patient characteristics are shown in Table 1.

\section{Pharmacological Treatment}

The substitutes, at the appropriate dose, were prescribed by doctors who were not part of the current study. Of the 105 participants, 69 patients received OAT therapy with MTD, 17 with BUP and 19 with SROM. The mean \pm SD dose per day of MTD was $97.3 \pm 67.1 \mathrm{mg}$, $10.1 \pm 8.5 \mathrm{mg}$ of BUP or $515.3 \pm 285.3 \mathrm{mg}$ of SROM. In patients with a recent change in opioid agonist medication or dose, the medication at the time of recruitment was used, and in case of doubt, the lower dose was chosen.

\section{Identification of Drug Consumption by Hair Analysis}

The hair analysis results showed significant differences between the 3 substitution medication groups in terms of additional heroin and cocaine consumption. If we only consider the presence or absence of heroin consumption, heroin co-use within a 3-month period was shown by hair testing in $62.3 \%$ of patients in the MTD group, in $23.5 \%$ of the BUP group and in $63.2 \%$ of the SROM group (Fig. 1). Thus, significantly fewer patients in the BUP group showed detection of heroin consumption compared to the MTD group (Pearson chi-square test, $\mathrm{X}^{2}$ $[1]=8.281, p<0.01$ ) and the SROM group (Pearson chisquare test, $\left.X^{2}[1]=5.707, p<0.05\right)$. By contrast, the MTD and SROM groups showed no significant differences (Pearson chi-square test, $\mathrm{X}^{2}[1]=0.004, p=0.947$ ). When we analysed heroin hair concentrations, we found that patients on BUP substitution consumed significantly less compared to patients taking MTD as a substitute (Kruskal-Wallis test, $\left.\mathrm{X}^{2}[1]=5.530, p<0.05\right)$. No other significant differences were found for either the MTD vs. SROM groups (Kruskal-Wallis test, $\mathrm{X}^{2}[1]=0.071$, $p=0.79$ ) or the SROM vs. BUP groups (Kruskal-Wallis test, $\mathrm{X}^{2}[1]=3.533, p=0.06$ ).

Cocaine consumption was detected in $79.7 \%$ of patients in the MTD group, in $64.7 \%$ of the BUP group 
Table 1. Patient characteristics

\begin{tabular}{|c|c|c|c|c|c|}
\hline Patient characteristics & $\begin{array}{l}\text { MTD } \\
(n=69)\end{array}$ & $\begin{array}{l}\text { BUP } \\
(n=17)\end{array}$ & $\begin{array}{l}\text { Morphine } \\
(n=19)\end{array}$ & $\begin{array}{l}\text { Total } \\
(n=105)\end{array}$ & $p$ value \\
\hline Gender, $n(\%)$ & & & & & $0.296^{1}$ \\
\hline Female & $21(30.4)$ & $2(11.8)$ & $5(26.3)$ & $28(26.7)$ & \\
\hline Age, years, mean $\pm S D$ & $41.15 \pm 8.1$ & $41.95 \pm 9.3$ & $39.68 \pm 10.8$ & $41.19 \pm 8.8$ & $0.714^{2}$ \\
\hline School, $n(\%)$ & & & & & $0.826^{1}$ \\
\hline Middle school & $25(36.2)$ & $5(29.4)$ & $5(26.3)$ & $35(33.3)$ & \\
\hline High school & $34(49.3)$ & $8(47.1)$ & $10(52.7)$ & $52(49.5)$ & \\
\hline Academic high school & $8(11.6)$ & $4(23.5)$ & $3(15.8)$ & $15(14.3)$ & \\
\hline Education, $n(\%)$ & & & & & $0.063^{1}$ \\
\hline None & $15(21.7)$ & $2(11.8)$ & $7(36.8)$ & $24(22.9)$ & \\
\hline Apprenticeship & $51(73.9)$ & $14(82.4)$ & $8(42.1)$ & $73(69.5)$ & \\
\hline Part-time & $6(9.0)$ & $0(0)$ & $1(5.6)$ & $7(7.0)$ & \\
\hline Unemployed & $8(11.9)$ & $2(13.3)$ & $0(0)$ & $10(10.0)$ & \\
\hline Disability & $38(56.7)$ & $5(33.3)$ & $14(77.8)$ & $57(57.0)$ & \\
\hline Student & $0(0)$ & $1(6.7)$ & $0(0)$ & $1(1.0)$ & \\
\hline Certified sick & $3(4.5)$ & $1(6.7)$ & $1(5.6)$ & $5(5.0)$ & \\
\hline Other & $9(13.4)$ & $3(20)$ & $1(5.6)$ & $13(13.0)$ & \\
\hline Number of psychiatric comorbidities, mean \pm SD & $1.62 \pm 1.1$ & $1.18 \pm 0.9$ & $2 \pm 1.1$ & $1.6 \pm 1.1$ & $0.497^{2}$ \\
\hline Number of clinical treatments, mean \pm SD & $6.68 \pm 8.2$ & $5.15 \pm 5.3$ & $5.96 \pm 6.7$ & $6.3 \pm 7.5$ & $0.748^{2}$ \\
\hline $\mathrm{BDI}$, mean $\pm \mathrm{SD}$ & $17.09 \pm 9.1$ & $14.12 \pm 9.2$ & $16.68 \pm 9.1$ & $16.51 \pm 9.1$ & $0.501^{2}$ \\
\hline $\mathrm{HIV}, n(\%)$ & & & & & $0.854^{1}$ \\
\hline Yes & $5(7.5)$ & $0(0)$ & $1(5.3)$ & $6(5.9)$ & \\
\hline No & $51(76.1)$ & $13(81.3)$ & $15(78.9)$ & $79(77.5)$ & \\
\hline
\end{tabular}

MTD, methadone; BUP, buprenorphine. ${ }^{1}$ Chi-square tests; ${ }^{2} t$ tests.

and in $84.2 \%$ of the SROM group. There were no significant differences between the 3 groups (MTD vs. BUP $p=0.19$, SROM vs. BUP $p=0.17$, MTD vs. SROM $p=0.66)$.

However, the patients in the BUP group used significantly lower doses of cocaine than the patients in the MTD group (Kruskal-Wallis test, $\mathrm{X}^{2}[1]=5.276, p<$ 0.05 ) and the SROM group (Kruskal-Wallis test, $X^{2}$ $[1]=4.093, p<0.05)$. There was no significant difference in the cocaine concentration in the MTD vs. SROM groups (Kruskal-Wallis test, $\mathrm{X}^{2}[1]=0.288$, $p=0.591)$.

There was a significant positive correlation between heroin and cocaine hair concentration (Spearman Rho correlation, two-tailed, $r=0.519, p<0.01$ ), indicating that high-dose users of heroin also show a high-dose use of cocaine. Heroin craving correlated with the MAM (heroin-use marker) hair concentration (Spearman Rho correlation, two-tailed, $r=0.406, p<0.01$ ), just as cocaine craving correlated with cocaine hair concentration (Spearman Rho correlations, two-tailed, $r=0.504$, $p<0.01$ ), confirming that high craving usually goes along with elevated drug co-consumption. In the case of BUP substitution, we found a significantly negative correlation between the dose of the medication and craving for heroin (Spearman Rho correlations, two-tailed, $r=-0.549$, $p<0.05)$, and a trend for negative correlation between the BUP medication dose and heroin co-consumption, (Spearman Rho correlations, two-tailed, $r=-0.469$, $p=0.058$. 


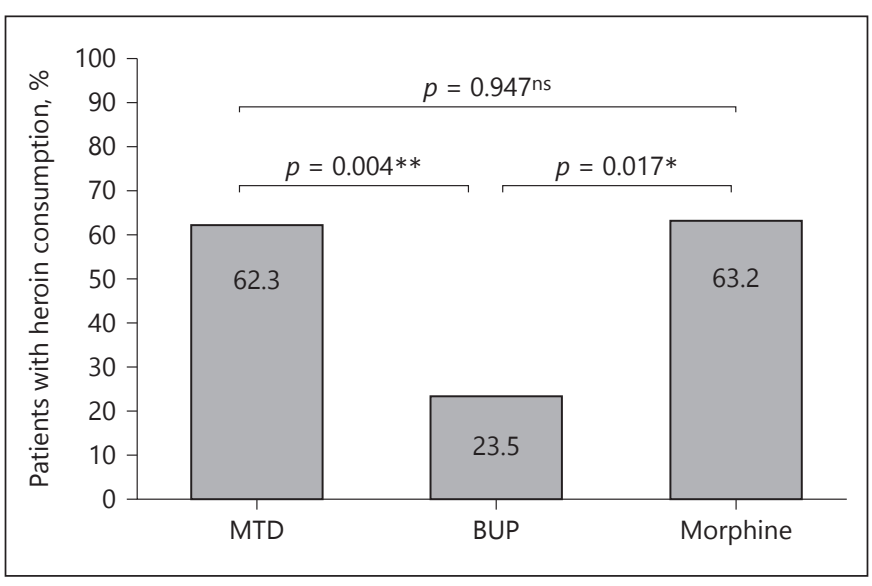

Fig. 1. Heroin consumption: percentage of patients with additional heroin consumption within the 3 substitution groups. MTD, methadone; BUP, buprenorphine; ns, non significant. ${ }^{*} p<0.05$; ** $p<0.01$.

\section{Discussion}

The results of this study suggest that substitution with BUP is associated with a lower level of additional heroin use compared to substitution with MTD or SROM. Moreover, the BUP-treated group also consumed a lower dose of cocaine compared to the MTD and SROM groups.

To the best of our knowledge, this is the first study to examine concomitant heroin and cocaine consumption in opioid-dependent patients in OAT using hair toxicology analysis. For the detection of drug use over a longer period of time, hair analysis is superior to the more commonly-used urine analysis $[29,30]$. The results of the hair analysis allowed us to compare, under daily practice conditions, the 3 substitution medications most commonly used in Switzerland for their effectiveness in reducing long-term concomitant heroin and cocaine consumption.

In this study, we found a negative correlation between the dose of BUP and heroin craving and consumption. This indicates that the dose of BUP in opioid agonist therapy should be high enough in order to receive an adequate therapeutic effect. We also detected a correlation between heroin and cocaine consumption. McCanceKatz et al. [31] have shown that chronic cocaine use decreases the BUP and MTD concentration in blood, with a potential reduction in response to treatment. Whether this effect also holds for SROM is not yet known, but this mechanism does represent a compelling rationale for the correlation between concomitant cocaine use and opioid agonist therapy failure in the form of increased heroin

Drug Use during Opioid Agonist Therapy consumption. BUP and SROM are non-inferior to MTD and are suitable alternatives to MTD for the OAT of heroin-dependent patients if MTD is not tolerated, if there is inadequate withdrawal suppression or a high illicit consumption of opioids.

Certain limitations should be mentioned. Screening was conducted in a single centre only and included a limited number of participants. This resulted in a lack of statistical power, particular in the BUP group. The naturalistic study design meant that all patients enrolled in this study were already under specific OAT and the assignment to the 3 different substitution medications was not randomized. This raises the possibility that allocation to the 3 treatment groups might have resulted in selection bias, particularly in view of the partial self-selection of patients to the different types of OAT medications that is common in Switzerland [22]. However, no significant differences between the 3 substitution groups were found regarding demographic variables such as age, gender, years and type of education, employment status, number of clinical treatments, number of psychiatric comorbidities, BDI score, or frequency of occurrence of HIV or Hepatitis C. Nevertheless, certain socioeconomic differences may have been overlooked due to the small total number of patients. Furthermore, the cross-sectional study design does not allow the inference of cause-effect relationships. The proportion of heroin or cocaine-positive hair samples in each substitution group and the quantitative measurement of heroin or cocaine by hair analysis was selected as the most important end-point because concomitant drug use was expected to be more relevant in this cross-over study than the retention-in-treatment rate. The influence of the different substitution medications on the retention rate was estimated to be relatively modest in ongoing maintenance treatment, and other studies have shown no difference in the retention-intreatment rate, or in the case of BUP only, in the first day of treatment (possibly because of a too low induction dose) $[19,32]$.

This study indicates that BUP is superior to MTD and SROM in terms of reducing illicit opioid and cocaine co-use. The cross-sectional character of the study means that no causal conclusions can be drawn and these findings need to be confirmed in a larger, randomized study. Further studies are needed to identify prognostic variables that allow optimal matching of patients to treatments, and the field of opioid agonist therapy in general needs to develop further in order to allow physicians to match the right OAT to the individual needs of a patient. 


\section{Statement of Ethics}

All participants provided written informed consent before admission to the study and were compensated with 5 Swiss Francs for their participation. The study protocol was approved by the local Ethics Committee of the canton of Zurich.

\section{Disclosure Statement}

M.H. received compensation for consultancy and a speaker's fee from Lundbeck. J.M. has received travel expenses and speaker fees from Lundbeck, Indivior and Takeda. B.B.Q. received a speaker's fee from Lundbeck. M.S. has worked as consultant and has received travel or research grants from Lundbeck, Indivior and Mundipharma.

\section{References}

1 Organization WH. Guidelines for the Psychosocially Assisted Pharmacological Treatment of Opioid Dependence. 2009.

2 Amato L, Minozzi S, Davoli M, Vecchi S. Psychosocial combined with agonist maintenance treatments versus agonist maintenance treatments alone for treatment of opioid dependence. Cochrane Database Syst Rev. 2011 Oct;(10):CD004147.

3 Ward J, Hall W, Mattick RP. Role of maintenance treatment in opioid dependence. Lancet. 1999 Jan;353(9148):221-6.

4 Bravo MJ, Llorens N, Barrio G, Brugal MT, Santos S, Sordo L, et al.; Itinere Project Group. Methadone maintenance treatment: a protective factor for cocaine injection in a street-recruited cohort of heroin users. Drug Alcohol Depend. 2010 Nov;112(1-2):62-8.

5 Herdener M, Dürsteler KM, Seifritz E, Nordt C. Changes in substance use in patients receiving opioid substitution therapy and resulting clinical challenges: a 17-year treatment case register analysis. Lancet Psychiatry. 2017 Apr;4(4):302-9.

6 Eurosurveillance editorial team. The European Monitoring Centre for Drugs and Drug Addiction publishes the European Drug Report 2013: trends and developments. Euro Surveill. 2013 May;18(22):20491.

7 Besson J, Beck T, Wiesbeck G, Hämmig R, Kuntz A, Abid S, et al. Opioid maintenance therapy in Switzerland: an overview of the Swiss IMPROVE study. Swiss Med Wkly. 2014 Mar;144:w13933.

8 Mattick RP, Breen C, Kimber J, Davoli M. Methadone maintenance therapy versus no opioid replacement therapy for opioid dependence. Cochrane Database Syst Rev. 2009 Jul;(3):CD002209.

9 Kastelic A, Dubajic G, Strbad E. Slow-release oral morphine for maintenance treatment of opioid addicts intolerant to methadone or with inadequate withdrawal suppression. Addiction. 2008 Nov;103(11):1837-46.

10 Kreek MJ. Medical safety and side effects of methadone in tolerant individuals. J Psychoactive Drugs. 1991;23(2):6 pages following 232.

11 Elkader A, Sproule B. Buprenorphine: clinical pharmacokinetics in the treatment of opioid dependence. Clin Pharmacokinet. 2005; 44(7):661-80
12 Amass L, Bickel WK, Crean JP, Blake J, Higgins ST. Alternate-day buprenorphine dosing is preferred to daily dosing by opioid-dependent humans. Psychopharmacology (Berl). 1998 Apr;136(3):217-25.

13 Fischer G, Gombas W, Eder H, Jagsch R, Peternell A, Stühlinger G, et al. Buprenorphine versus methadone maintenance for the treatment of opioid dependence. Addiction. 1999 Sep;94(9):1337-47.

14 Giacomuzzi SM, Riemer Y, Ertl M, Kemmler G, Rössler H, Hinterhuber H, et al. Buprenorphine versus methadone maintenance treatment in an ambulant setting: a health-related quality of life assessment. Addiction. 2003 May;98(5):693-702.

15 Montoya ID, Gorelick DA, Preston KL, Schroeder JR, Umbricht A, Cheskin LJ, et al. Randomized trial of buprenorphine for treatment of concurrent opiate and cocaine dependence. Clin Pharmacol Ther. 2004 Jan; 75(1):34-48.

16 Gerra G, Borella F, Zaimovic A, Moi G, Bussandri $\mathrm{M}$, Bubici $\mathrm{C}$, et al. Buprenorphine versus methadone for opioid dependence: predictor variables for treatment outcome. Drug Alcohol Depend. 2004 Jul;75(1):37-45.

17 Eder H, Jagsch R, Kraigher D, Primorac A, Ebner N, Fischer G. Comparative study of the effectiveness of slow-release morphine and methadone for opioid maintenance therapy. Addiction. 2005 Aug;100(8):1101-9.

18 Hämmig R, Köhler W, Bonorden-Kleij K, Weber B, Lebentrau K, Berthel T, et al. Safety and tolerability of slow-release oral morphine versus methadone in the treatment of opioid dependence. J Subst Abuse Treat. 2014 Oct; 47(4):275-81.

19 Jegu J, Gallini A, Soler P, Montastruc JL, Lapeyre-Mestre M. Slow-release oral morphine for opioid maintenance treatment: a systematic review. Br J Clin Pharmacol. 2011 Jun; 71(6):832-43.

20 Beck T, Haasen C, Verthein U, Walcher S, Schuler C, Backmund M, et al. Maintenance treatment for opioid dependence with slow-release oral morphine: a randomized cross-over, non-inferiority study versus methadone. Addiction. 2014 Apr; 109(4): $617-26$.

21 Mutschler J, Gastberger S, Baumgartner MR, Grosshans M, Seifritz E, Quednow BB, et al.
Pregabalin Use Among Opioid-Addicted Patients in Switzerland. J Clin Psychiatry. 2016 Sep;77(9):1202-3.

22 Nordt C, Vogel M, Dey M, Moldovanyi A, Beck T, Berthel T, et al. One size does not fit all-evolution of opioid agonist treatments in a naturalistic setting over 23 years. Addiction. 2019 Jan;114(1):103-11.

23 Beck AT, Ward CH, Mendelson M, Mock J, Erbaugh J. An inventory for measuring depression. Arch Gen Psychiatry. 1961 Jun;4(6): $561-71$.

24 Spielberger CD, Gorsuch R. L., Lushene, R., Vagg, P. R., \& Jacobs, G. A. Manual for the State-Trait Anxiety Inventory. Palo Alto (CA): Consulting Psychologists Press; 1983.

25 Rosenberg H. Clinical and laboratory assessment of the subjective experience of drug craving. Clin Psychol Rev. 2009 Aug;29(6): 519-34.

26 Madry MM, Bosshard MM, Kraemer T, Baumgartner MR. Hair analysis for opiates: hydromorphone and hydrocodone as indicators of heroin use. Bioanalysis. 2016 May;8(9): 953-64.

27 Pötsch L, Skopp G. Stability of opiates in hair fibers after exposure to cosmetic treatment. Forensic Sci Int. 1996 Aug;81(2-3): 95-102.

28 Rust KY, Baumgartner MR, Meggiolaro N, Kraemer T. Detection and validated quantification of 21 benzodiazepines and 3 " $\mathrm{z}$-drugs" in human hair by LC-MS/MS. Forensic Sci Int. 2012 Feb;215(1-3):64-72.

29 Pragst F, Balikova MA. State of the art in hair analysis for detection of drug and alcohol abuse. Clin Chim Acta. 2006 Aug;370(1-2): 17-49.

30 Hegstad S, Khiabani HZ, Kristoffersen L, Kunøe N, Lobmaier PP, Christophersen AS. Drug screening of hair by liquid chromatography-tandem mass spectrometry. J Anal Toxicol. 2008 Jun;32(5):364-72.

31 McCance-Katz EF, Jatlow P, Rainey PM. Effect of cocaine use on methadone pharmacokinetics in humans. Am J Addict. 2010 JanFeb;19(1):47-52.

32 Petitjean S, Stohler R, Déglon JJ, Livoti S, Waldvogel D, Uehlinger C, et al. Doubleblind randomized trial of buprenorphine and methadone in opiate dependence. Drug Alcohol Depend. 2001 Mar;62(1):97-104. 